ran, die Kosten der Wahlniederlage zu minimieren. ${ }^{30}$ Im deutschen gemischten Wahlsystem kommt als Besonderheit hinzu, dass auch der Wahlerfolg sich sehr ungleich in den beiden Wahlrechtskomponenten niederschlägt. Man kann daher sagen, dass sich die beiden großen Volksparteien durch ihre doppelten Nominierungsstrategien auch vor den Kosten des eigenen Wahlerfolgs schützen müssen, wenn ein überproportional hoher Zugewinn an Direktmandaten auf Kosten der eigenen Listenkandidaturen zu gehen droht. Die KartellparteiThese ist bislang nicht im Hinblick auf die Nominierungsstrategien von Parteien empirisch überprüft worden. Schotten sie sich wirklich zunehmend gegenüber dem Wähler ab? Unser Befund der häufiger werdenden Kombination guter Listenplätze mit aussichtsreichen Wahlkreisen lässt sich durchaus als Bestätigung dieser Abschottungshypothese verstehen. Dennoch ist nicht davon auszugehen, dass diese privilegierte, weil doppelt abgesicherte Abgeordnetengruppe sich deswegen ausschließlich an der Partei orientiert, denn aktive Wahlkreisarbeit gehört, gerade auch aufgrund der Kontaminationseffekte in gemischten Wahlsystemen, zu den zentralen Leistungskriterien eines Abgeordneten, die Parteien bei der Wiedernominierung dieser Doppelkandidaten - und das heißt auch bei ihrer aussichtsreichen Platzierung auf den Listen - anlegen. Insofern wäre eine weitere Annahme der Kartellpartei-These, dass sich die Parteien durch ihre Immunisierung gegenüber elektoraler Unsicherheit zunehmend aus der Gesellschaft wegbewegen, mit den vorliegenden Ergebnissen unserer Studie nicht zu belegen.

30 Vgl. Richard S. Katz / Peter Mair, Changing Models of Party Organizations and Party Democracy, a.a.O. (Fn. 2); dies., The Cartel Party Thesis, a.a.O. (Fn. 2).

\title{
Überhangmandate bei der Bundestagswahl 2009. Eine Schätzung mit Simulationen
}

\author{
Joachim Behnke
}

Eine der in der öffentlichen Wahrnehmung problematischsten Konsequenzen der Bundestagswahl im September 2009 könnte darin bestehen, dass sich die neue Regierung auf eine Mehrheit im Parlament stützen würde, die lediglich durch die so genannten Überhangmandate garantiert wäre. Überhangmandate entstehen, wenn eine Partei in einem Bundesland aufgrund der Erststimmen mehr Direktmandate gewinnt, als ihr dort nach der proportionalen Zuteilung der Sitze anhand der Zweitstimmen zustehen würden. Sie sind eine Merkwürdigkeit und die wohl am wenigsten verstandene Komponente des deutschen Wahlsystems, das auch ansonsten nicht gerade an einem Mangel an Komplexität leidet. Fehlendes Verständnis erstreckt sich dabei nicht nur auf die Bürger, sondern ebenso auf die überwiegende Anzahl von Politikern und Journalisten.

Überhangmandate können niemals einen gewollten und wünschenswerten Effekt darstellen, sind bestenfalls ein hinzunehmendes vernachlässigbares Übel. Im schlimmsten Fall aber untergraben sie das allem anderen übergeordnete Ziel eines Wahlsystems in der parlamentarischen Demokratie: die Legitimation des Parlaments und der von ihm gewählten 
Regierung. Damit diese Entscheidungen treffen können, die allgemeinverbindlich sind und deren Gültigkeit von allen akzeptiert wird, muss das Verfahren unstrittig sein, mit dem sie dazu ermächtigt werden.

Dass der Gesetzgeber trotz der möglichen gravierenden Konsequenzen der Überhangmandate dieses Thema in den letzten Jahren höchst nachlässig behandelt hat, ist leicht zu erklären. Überhangmandate haben bisher immer die Koalition begünstigt, die die Regierung bildete, auch wenn dies rein theoretisch nicht zwangsläufig der Fall sein muss. Diejenigen, die durch einfaches Gesetz das Wahlrecht ändern könnten, sind also die von ihr Begünstigten. Als 1994 die CDU/FDP-Koalition durch insgesamt zwölf Überhangmandate der CDU ihren Vorsprung gegenüber der Opposition von zwei auf zehn Sitze ausbauen konnte, da die SPD lediglich vier Überhangmandate erhalten hatte, sah sich die SPD, in Gestalt der niedersächsischen Landesregierung unter Gerhard Schröder, genötigt, eine Klage beim Bundesverfassungsgericht anzustrengen. Das Verfahren endete mit dem Urteil vom 10. April 1997 in einem Patt von 4:4 Stimmen, womit die alte Regelung bestehen blieb. Als jedoch 1998 die SPD zusammen mit Bündnis 90 / Die Grünen die Mehrheit im Bundestag erlangte und nun selbst vom Gewinn von 13 Überhangmandaten profitierte, ließ sie weder unmittelbar nach der Wahl noch in den folgenden sieben Jahren irgendeinen Ehrgeiz erkennen, die ihr nun zugewachsene Möglichkeit zu nutzen, die Überhangmandate abzuschaffen. Aus denselben machtpolitischen Überlegungen heraus ist leicht zu verstehen, dass Grüne, Linke und Sozialdemokraten vor der Sommerpause 2009 auf eine rasche Abschaffung der Überhangmandate noch vor der Bundestagswahl im September drängten, während CDU und FDP keinen akuten Handlungsbedarf erkennen konnten. Denn es spricht einiges dafür, dass die Überhangmandate bei der Bundestagswahl 2009 vor allem der CDU zugute kommen und so das Polster an Mandaten einer schwarz-gelben Koalition verstärken könnten.

Dabei hatten diejenigen, die für eine eilige Änderung des Wahlgesetzes eintraten, Rückenwind durch das Bundesverfassungsgerichtsurteil vom 3. Juli 2008, durch das das derzeitige Wahlrecht als in Teilen verfassungswidrig und eine diesbezügliche Änderung als erforderlich erklärt worden war. Hierfür setzte das Gericht dem Gesetzgeber eine Frist bis spätestens 30. Juni 2011. Auslöser des Verfassungsgerichtsurteils war das Phänomen des so genannten „negativen Stimmgewichts“, das bei der Nachwahl in einem Dresdner Wahlkreis dazu geführt hatte, dass die CDU durch mehr Zweitstimmen weniger Sitze erhalten hätte. Das negative Stimmgewicht stellt also eine spezifische Form der Entstehung von Überhangmandaten dar. Doch auch wenn es immer in Zusammenhang mit einem Überhangmandat auftritt, so gilt dies umgekehrt keineswegs. Die überwiegende Anzahl der Überhangmandate ist auf andere Faktoren wie zum Beispiel die Wahlkreiseinteilung, schwankende Wahlbeteiligung in den Bundesländern, die Größe der Dritt- und Viertparteien oder Stimmensplitting zurückzuführen. Die Beseitigung der Überhangmandate würde also automatisch den Effekt des negativen Stimmgewichts auslöschen, umgekehrt lassen sich aber sehr wohl Möglichkeiten denken, die das negative Stimmgewicht beseitigen, ohne die Überhangmandate anzutasten. So lassen sich auch die Differenzen zwischen den Fraktionen erklären, die bei der abschließenden Diskussion über den Gesetzentwurf der Grünen zur Änderung des Wahlgesetzes am 3. Juli 2009 im Bundestag hervorgetreten sind. Ihr Vorschlag sah vor, dass einer Partei Überhangmandate in Form von Listenmandaten in Ländern, in denen keine Überhangmandate entstehen, abgezogen würden. Damit wären Überhangmandate vermutlich mehr oder weniger abgeschafft worden, mit Ausnahme jener, die für die CSU anfallen 
würden, da diese ja nicht mit anderen Landeslisten verrechnet werden könnten. In der Debatte wurde jedoch deutlich, dass die Union offensichtlich mit einer Lösung liebäugelt, die das negative Stimmgewicht beseitigen würde, ohne die Überhangmandate wesentlich zu berühren, so zum Beispiel durch eine Trennung der Landeslisten. Da diese unterschiedlichen Ansichten, die letztlich wohl auch unterschiedliche Interessen widerspiegeln, zur Ablehnung des Entwurfs der Grünen führten, wird die Bundestagswahl 2009 nach dem alten Wahlgesetz durchgeführt. Es werden also weiterhin Überhangmandate entstehen, die nicht kompensiert werden.

Lange Zeit fielen Überhangmandate so selten an, dass sie - für Politik wie Wissenschaft - ein vernachlässigbarer Faktor waren, zumindest in Hinsicht auf ihre realen Konsequenzen. Die Situation änderte sich schlagartig mit der deutschen Wiedervereinigung. Seit 1990 ist die Zahl der Überhangmandate sprunghaft angestiegen, was vor allem damit zusammenhängt, dass die neuen Bundesländer aus mehreren Gründen eine besondere Anfälligkeit für ihre Entstehung aufweisen. ${ }^{1}$ Die Anzahl der Überhangmandate erreichte 1994 und 2005 mit jeweils 16 ihren höchsten Wert; der größte Vorteil für eine Partei entstand 1998, als die SPD alle 13 Überhangmandate für sich allein verbuchen konnte. Es spricht alles dafür, dass die Bundestagswahl 2009 für einen neuen Rekord an Überhangmandaten sorgen könnte. Sollte sich der von durchweg allen Umfragen ermittelte desaströse Einbruch der SPD am Wahltag in entsprechendem Ausmaß verwirklichen, würde dies mit hoher Wahrscheinlichkeit zu einer bisher nicht dagewesenen Anzahl von Überhangmandaten führen, mit entsprechenden Folgen für die Möglichkeiten der Koalitionsbildung.

Im Folgenden sollen diese möglichen Konsequenzen der Überhangmandate mit Hilfe einer Reihe von Simulationen abgeschätzt werden. Dabei geht es nicht um eine genaue Punktprognose des Ergebnisses; vielmehr sind die zugrunde liegenden Annahmen bewusst so einfach und grob gehalten, dass die Simulation in erster Linie die Bandbreite der möglichen Resultate und deren Abhängigkeit von bestimmten strategischen Verhaltensformen der Anhänger der verschiedenen Parteien aufzeigen soll. Die Methode der Simulation scheint für den verfolgten Zweck besonders geeignet, da auf diese Weise die Prognosen und Schätzungen grundsätzlich inhärente Unsicherheit berücksichtigt werden kann. Im Gegensatz zu den verbreiteten ex-post beziehungsweise statischen Berechnungen, die einem Wahlergebnis eine entsprechende Sitzverteilung zuweisen ${ }^{2}$, werden durch die hier durchgeführte Simulation einer bestimmten Stimmenverteilung verschiedene Sitzverteilungen zugeordnet, die die Unsicherheit hinsichtlich der angenommenen Stimmenverteilung widerspiegeln.

1 Vgl. Florian Grotz, Die personalisierte Verhältniswahl unter den Bedingungen des gesamtdeutschen Parteiensystems. Eine Analyse der Entstehungsursachen von Überhangmandaten seit der Wiedervereinigung, in: PVS, 41. Jg. (2000), S. 707 - 729; Joachim Behnke, Ein integrales Modell der Ursachen von Überhangmandaten, in: PVS, 44. Jg. (2003), S. 41 - 65; ders., Überhangmandate und Parteienstruktur, in: Frank Brettschneider / Jan van Deth / Edeltraud Roller (Hrsg.), Die Bundestagswahl 2002, Wiesbaden 2004, S. 327 - 352.

2 Vgl. zum Beispiel Christian Rindsfüßer / Susanne Schäfer-Walkmann, Wahlarithmetische Kabinettsstückchen: Mandate im Überfluß oder wie Überhangmandate die Wahl entscheiden, in: ZA-Informationen, 1998, H. 43, S. 124 - 134; Joachim Behnke, Das Wahlsystem der Bundesrepublik Deutschland. Logik, Technik und Praxis der Verhältniswahl, Baden-Baden 2007, S. 215 ff.; Ossip Fürnberg / Danko Knothe, Wahlsiege ohne Stimmenmehrheit: Auswirkungen von verstärktem „Lagersplitting“ auf Mandatsverteilung und Koalitionsoptionen, in: ZParl, 40. Jg. (2009), H. 1, S. $56-74$. 
Eine bestimmte durch Umfragen prognostizierte Stimmenverteilung führt also nicht zu einem bestimmten Ergebnis mit den entsprechenden Koalitionsbildungsmöglichkeiten, sondern zu einer Vielfalt von einzelnen Ergebnissen, die jeweils mit einer bestimmten Wahrscheinlichkeit auftreten. Dies hat unter anderem den Vorteil, dass bezüglich einer konkreten Stimmenverteilung einer Umfrage auch die Wahrscheinlichkeit angegeben werden kann, mit der sich aus diesen Daten eine bestimmte Koalitionsmöglichkeit ergibt. Die Stärke des Einflusses bestimmter Formen strategischen Verhaltens kann dann als Veränderung dieser Wahrscheinlichkeiten wesentlich differenzierter ausgedrückt werden, als es bei einer statischen Vorgehensweise der Fall wäre, die nur die diskreten Zustände ja/nein kennt.

\section{Das Grundmodell der Simulation}

Als Ausgangsdatenbasis dienen der Simulation die Ergebnisse der Bundestagswahl von 2005 in allen 299 Wahlkreisen. In einem ersten Schritt werden diese Daten dem allgemeinen, das heißt mittleren Trend der Parteien, wie er sich in Umfragen widerspiegelt, angepasst. Als Quellen wurden hierzu folgende Umfragen herangezogen: Politbarometer vom 3. Juli, Infratest-dimap vom 2. Juli, EMNID vom 9. Juli und Forsa vom 8. Juli 2009. Das Ergebnis der CDU weist einen leichten Trend nach oben von einem zusätzlichen Prozentpunkt auf. Leider ist es nicht möglich, den Umfragen die einzelnen Werte für CDU und CSU zu entnehmen. Die Schätzung für die CSU beruht daher auf mehr oder weniger plausiblen Überlegungen. Auch wenn die Landtagswahl 2008 mit einem Verlust von 19 Prozentpunkten nicht repräsentativ für die derzeitige Lage der CSU sein dürfte, bestätigte auch die Wahl zum Europäischen Parlament, dass die Zeiten vorbei sind, in denen die CSU die Formel „50+x“ nach außen als Wahlziel proklamieren konnte, ohne das Risiko eines Gesichtsverlusts einzugehen. Zwei Prozentpunkte weniger an Stimmen scheinen daher ein nicht zu hoch gegriffener und vorsichtiger Schätzwert für die CSU zu sein. Dramatisch fallen die Umfrageergebnisse bekanntlich für die SPD aus. Sie liegt im Juli 2009 im Mittel bei circa 24 Prozent, ihr Verlust gegenüber 2005 wird daher auf zehn Punkte veranschlagt. Die drei kleinen Parteien legen alle leicht zu, die FDP und Die Grünen vier Prozentpunkte, Die Linke einen Punkt.

Der in den Umfragen ermittelte Trend wird als in allen Wahlkreisen mehr oder weniger homogen vorhanden angenommen. ${ }^{3}$ Dies scheint eine zulässige und aufgrund der Datenlage auch notwendige Vereinfachung, da die Umfrageergebnisse keine Rückschlüsse auf die Landesebene zulassen. Die von dem einen oder anderen Umfrageinstitut getrennten Daten für Ost und West ermitteln außerdem zwar die bekannten und erwarteten Niveauunterschiede, differieren aber nicht bedeutsam hinsichtlich des Trends. Auch werden die diversen

3 Natürlich sind auch genauere Prognosen auf der Ebene der einzelnen Wahlkreise möglich, wenn man zusätzliche Kriterien wie Amtsinhaberschaft und Geschlecht der Kandidaten für den Wahlkreis berücksichtigt (vgl. Christian Mackenrodt, Wer gewinnt das Direktmandat? Prognosemodelle für das Erststimmenergebnis in den Wahlkreisen bei Bundestagswahlen, unveröffentlichte Diplomarbeit, Bamberg 2007). Aber auch hier gilt, dass mit der Annahme des homogenen Trends über alle Wahlkreise zwar die Ergebnisse in den einzelnen Wahlkreisen nicht mit derselben Treffergenauigkeit vorausgesagt werden können, die Gesamtverteilung sich aber kaum durch die Hinzunahme dieser zusätzlichen Informationen verändern würde. 
Landtagswahlen nicht in die Trendschätzung einbezogen, da sie als so genannte Nebenoder Zwischenwahlen oft einer anderen Dynamik gehorchen als Bundestagswahlen.

Für die Simulation werden insgesamt für jedes Modell 1000 Iterationen durchgeführt. ${ }^{4}$ In einem ersten Schritt werden die Zweitstimmenergebnisse in den Wahlkreisen bestimmt. Um die Unsicherheiten von Umfrageergebnissen zu berücksichtigen, wird angenommen, dass der "tatsächliche“ Trend mit einer Standardabweichung von 2,0 Prozentpunkten bei den großen Parteien und 1,5 Punkten bei den kleinen Parteien um den globalen Trend normalverteilt ist. Für jede einzelne Iteration wird also der realisierte Wert einer dementsprechenden Zufallsvariable dem "globalen "Trend hinzugefügt. ${ }^{5}$ Die Ergebnisse in den einzelnen Wahlkreisen werden wiederum so simuliert, dass der dortige Trendfaktor ebenfalls zufällig um den iterationsspezifischen Trendfaktor mit einer Standardabweichung von drei Prozentpunkten bei CDU/CSU und bei der Partei Die Linke in den neuen Bundesländern (inklusive Berlin), bei der SPD mit einer Standardabweichung von vier Punkten und bei den kleinen Parteien FDP, Bündnis 90 / Die Grünen und Die Linke in den alten Bundesländern mit einer Standardabweichung von zwei Prozentpunkten streut. ${ }^{6}$

In einem zweiten Schritt werden nun die wahlkreisspezifischen Erststimmenergebnisse geschätzt. Bekanntlich liegen diese bei den großen Parteien aufgrund des Stimmensplittings deutlich über den Zweitstimmenergebnissen. Die Erststimmen von CDU und SPD für die einzelnen Wahlkreise wurden daher geschätzt, indem den Zweitstimmen jeweils 60 Prozent der Zweitstimmen des kleinen Wunschkoalitionspartners hinzugezählt wurden, bei der CDU also die der FDP, bei der SPD die der Grünen. Dies entspricht ungefähr der Größenordnung des Stimmensplittings, wie es bei der letzten Bundestagswahl praktiziert worden ist. 2005 gaben 57 Prozent der Wähler, die mit der Zweitstimme für die Grünen votiert

4 Für eine Simulation scheinen 1.000 Iterationen nicht sehr viel zu sein, da hier die Erhöhung der Fallzahl im Gegensatz zu Umfragen ja keine weiteren Kosten verursacht. Tests mit 10.000 Iterationen zeigen jedoch, dass sich die Ergebnisse nur unwesentlich ändern, da die Mittelwerte der interessierenden Variablen sehr schnell konvergieren, so dass aus Gründen der Praktikabilität die $1.000 \mathrm{er}-$ Fallzahl vorzuziehen ist.

5 Diese Zufallsvariablen sind jeweils unabhängig voneinander. Eine positive Abweichung einer Partei vom globalen Trend korreliert also nicht mit den Abweichungen der anderen Parteien. Tatsächlich lassen sich für die Wahlkreisergebnisse der Parteien in den letzten beiden Wahlen 2002 und 2005 auch keine „Fehlerkorrelationen“ feststellen, Gewinne einer Partei hängen nicht systematisch mit den Gewinnen oder Verlusten einer bestimmten anderen Partei zusammen. Allerdings müssen sich die Gesamtgewinne und -verluste natürlich zu Null addieren. Da dies hier nicht explizit modelliert worden ist, wird diese Kompensation also zum Teil zumindest in die Restkategorien der sonstigen Parteien verschoben. Ansonsten führt die Nichtberücksichtigung zu einer leichten Unterschätzung der Unterschiede, also zu einer geringfügigen Überbewertung der Mitte, dem globalen Trend.

6 Während sich die Standardabweichungen des bundesweiten Trendfaktors grob an den Standardfehlern von den üblichen 1.000er-Stichproben orientieren (die in Wirklichkeit eher noch größer sein dürften aufgrund von Fehlern bei der Stichprobenziehung, worauf auch die Differenzen zwischen den Instituten hinweisen), orientiert sich die iterationsinterne Standardabweichung zwischen den Wahlkreisen an den Streuungen der Differenzen der Wahlkreisergebnisse zwischen 2002 und 2005. Tatsächlich lagen diese empirischen Streuungen alle (mit Ausnahme der Ergebnisse der SPD) unter drei Prozentpunkten. Aus diesem Grund wurde für die SPD eine etwas höhere Streuung angenommen. Dies ist insofern zu vertreten, als die interne Streuung auch mit der Größe der Veränderung zu korrelieren scheint. Parteien, deren Anteil zwischen zwei Wahlen relativ stabil ist, streuen auch weniger zwischen den Wahlkreisen hinsichtlich der Veränderung zwischen zwei Wahlen. 
hatten, ihre Erststimme der SPD, und 52 Prozent Zweitstimmen-FDP-Wähler entschieden sich, mit der Erststimme CDU zu wählen. ${ }^{7}$ Da die FDP sich 2009 deutlich klarer als 2005 für eine schwarz-gelbe Koalition ausgesprochen hat, dürfte der Anteil der im Sinne dieser Wunschkoalition taktischen Splitter unter den FDP-Anhängern 2009 wohl höher ausfallen als 2005, die veranschlagten 60 Prozent könnten daher sogar unterschätzt sein. Auch beim

\begin{tabular}{|c|c|c|c|c|c|c|c|}
\hline \multicolumn{8}{|c|}{$\begin{array}{l}\text { Tabelle 1a: Stimmensplittingmuster zugunsten der SPD für Wähler, die mit der Zweitstimme } \\
\text { Die Grünen beziehungsweise Die Linke wählen, nach Bundesländern }\end{array}$} \\
\hline \multirow{2}{*}{ Land } & \multirow{2}{*}{ 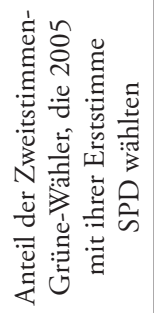 } & \multirow{2}{*}{ 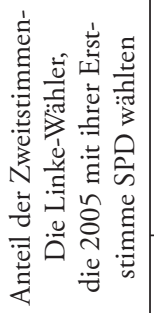 } & \multicolumn{5}{|c|}{$\begin{array}{l}\text { Überhangmandate für die SPD } \\
\text { bei vergangenen Bundestagswahlen }\end{array}$} \\
\hline & & & 1990 & 1994 & 1998 & 2002 & $\begin{array}{c}1990 \text { bis } \\
2002\end{array}$ \\
\hline Baden-Württemberg & 57,53 & 23,20 & 0 & 0 & 0 & 0 & 0 \\
\hline Bayern & 48,75 & 18,99 & 0 & 0 & 0 & 0 & 0 \\
\hline Berlin & 42,56 & 21,02 & 0 & 0 & 0 & 0 & 0 \\
\hline Brandenburg & 61,90 & 13,26 & 0 & 3 & 3 & 0 & 6 \\
\hline Bremen & 53,80 & 20,54 & 0 & 1 & 0 & 0 & 1 \\
\hline Hamburg & 58,25 & 27,09 & 0 & 0 & 1 & 1 & 2 \\
\hline Hessen & 62,80 & 26,72 & 0 & 0 & 0 & 0 & 0 \\
\hline Mecklenburg-Vorpommern & 49,83 & 11,96 & 0 & 0 & 2 & 0 & 2 \\
\hline Niedersachsen & 62,34 & 24,17 & 0 & 0 & 0 & 0 & 0 \\
\hline Nordrhein-Westfalen & 60,06 & 25,33 & 0 & 0 & 0 & 0 & 0 \\
\hline Rheinland-Pfalz & 59,12 & 22,36 & 0 & 0 & 0 & 0 & 0 \\
\hline Saarland & 59,35 & 20,46 & 0 & 0 & 0 & 0 & 0 \\
\hline Sachsen & 45,52 & 9,61 & 0 & 0 & 0 & 0 & 0 \\
\hline Sachsen-Anhalt & 58,71 & 12,90 & 0 & 0 & 4 & 2 & 6 \\
\hline Schleswig-Holstein & 66,29 & 31,79 & 0 & 0 & 0 & 0 & 0 \\
\hline Thüringen & 51,85 & 13,31 & 0 & 0 & 3 & 1 & 4 \\
\hline
\end{tabular}

Splittingverhalten wird ein homogenes Muster für alle Wahlkreise unterstellt. Zumindest auf der Landesebene lassen sich in der Tat keine allzu großen Unterschiede erkennen, wie in den Tabellen 1a und $1 \mathrm{~b}$ zu erkennen ist. Auffällige Abweichungen zeigen sich allenfalls in Berlin. Die relativ geringen Unterschiede sind insofern bemerkenswert, als sie belegen, dass es offensichtlich keinen Lerneffekt dergestalt gibt, dass das Splittingverhalten zunimmt,

7 Die Zahlen über das bundesweite Splittingverhalten stammen aus der so genannten Repräsentativen Wahlstatistik und sind enthalten in: Doreen Namislo / Karina Schorn / Margitta von Schwartzenberg, Wählerverhalten bei der Bundestagswahl 2005 nach Geschlecht und Alter, in: Wirtschaft und Statistik, 3/2006, S. 220 - 237, S. 234. 


\begin{tabular}{|c|c|c|c|c|c|c|}
\hline \multicolumn{7}{|c|}{$\begin{array}{l}\text { Tabelle 1b: Stimmensplittingmuster zugunsten der CDU für die Wähler, die mit der Zweitstimme } \\
\text { FDP wählen, nach Bundesländern }\end{array}$} \\
\hline \multirow{2}{*}{ Land } & \multirow{2}{*}{ 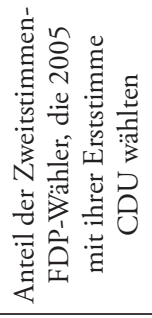 } & \multicolumn{5}{|c|}{$\begin{array}{l}\text { Überhangmandate für die CDU } \\
\text { bei den vergangenen Bundestagswahlen }\end{array}$} \\
\hline & & 1990 & 1994 & 1998 & 2002 & $\begin{array}{c}1990 \mathrm{bi} \\
2002\end{array}$ \\
\hline Baden-Württemberg & 64,39 & 0 & 2 & 0 & 0 & 2 \\
\hline Bayern & 60,05 & 0 & 0 & 0 & 0 & 0 \\
\hline Berlin & 20,58 & 0 & 0 & 0 & 0 & 0 \\
\hline Brandenburg & 48,55 & 0 & 0 & 0 & 0 & 0 \\
\hline Bremen & 56,50 & 0 & 0 & 0 & 0 & 0 \\
\hline Hamburg & 64,86 & 0 & 0 & 0 & 0 & 0 \\
\hline Hessen & 62,29 & 0 & 0 & 0 & 0 & 0 \\
\hline Mecklenburg-Vorpommern & 50,98 & 2 & 2 & 0 & 0 & 4 \\
\hline Niedersachsen & 63,03 & 0 & 0 & 0 & 0 & 0 \\
\hline Nordrhein-Westfalen & 60,78 & 0 & 0 & 0 & 0 & 0 \\
\hline Rheinland-Pfalz & 59,17 & 0 & 0 & 0 & 0 & 0 \\
\hline Saarland & 56,33 & 0 & 0 & 0 & 0 & 0 \\
\hline Sachsen & 50,83 & 0 & 3 & 0 & 1 & 4 \\
\hline Sachsen-Anhalt & 50,26 & 3 & 2 & 0 & 0 & 5 \\
\hline Schleswig-Holstein & 67,28 & 0 & 0 & 0 & 0 & 0 \\
\hline Thüringen & 49,12 & 1 & 3 & 0 & 0 & 4 \\
\hline
\end{tabular}

wenn es sich als taugliches Mittel erwiesen hat, um zusätzliche Überhangmandate zu erzielen. Auch in den Bundesländern, die definitiv keine Kandidaten für Überhangmandate darstellen, treten die bekannten Splittingmuster in vergleichbarer Größe auf.

Etwas komplizierter liegt der Fall bei der Partei Die Linke. Ihre Zweitstimmen-Wähler entschieden sich 2005 zu 17 Prozent mit der Erststimme für die SPD. Hier sollte jedoch die natürliche Neigung, sich strategisch zu verhalten, eher abgenommen haben, da Die Linke zumindest in den neuen Bundesländern ernsthaft mit den anderen großen Parteien um Direktmandate konkurriert. Für die Wähler, die ihre Zweitstimme den Linken geben, wird daher im Standardmodell angenommen, dass sie in den neuen Bundesländern und in Berlin auch mit der Erststimme Die Linke wählen, also ein so genanntes „straight ticket“ abgeben, während ihr Anteil im Westen, wo keine Aussichten auf den Gewinn von Direktmandaten bestehen, im Standardmodell auf 25 Prozent gesetzt wird. Alle anderen außer den genannten Splittingmustern werden ignoriert, da sie nur in einem vernachlässigbaren Umfang auftreten. Die einzige Ausnahme stellen möglicherweise FDP-Anhänger dar, die eine sozialliberale Koalition bevorzugen würden. So haben 2005 immerhin sieben Prozent der Zweitstimmen-FDP-Wähler ihre Erststimme der SPD gegeben. Aufgrund der klaren 
Koalitionsaussage 2009 kann jedoch davon ausgegangen werden, dass dieser Anteil dieses Mal wesentlich geringer ausfallen wird und daher unberücksichtigt bleiben kann. Zusammenfassend sind alle Modellannahmen in Tabelle 2 wiedergegeben.

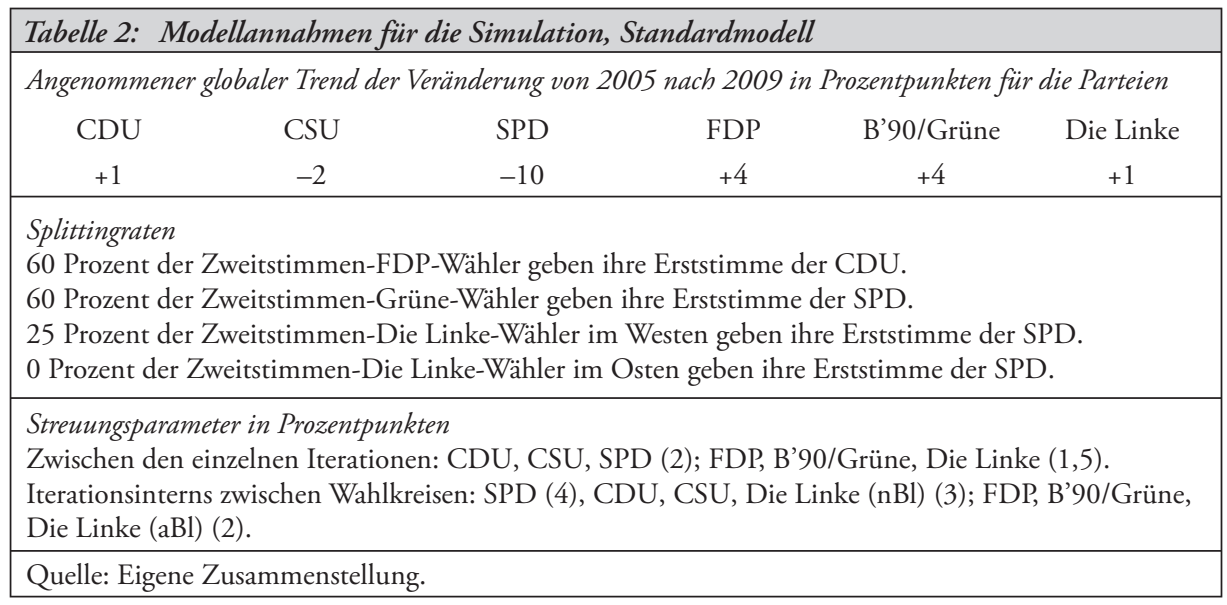

Entsprechend den so simulierten Erststimmenzahlen aller Parteien in den Wahlkreisen wird für jeden Wahlkreis der Gewinner des Direktmandats ermittelt. Anschließend werden die Direktmandate und die Zweitstimmen auf der Landesebene aggregiert und die Sitze analog zu echten Wahlen nach dem Sainte-Laguë-Verfahren, das bei der kommenden Bundestagswahl zum ersten Mal Verwendung findet, verteilt. Die Simulationen des Standardmodells ergaben die folgenden Ergebnisse:

\begin{tabular}{|c|c|c|c|c|c|}
\hline \multicolumn{7}{|c|}{ Tabelle 3: Wesentliche Ergebnisse der Simulation (Standardmodell) } \\
\hline \multicolumn{7}{|c|}{ Mittlere Anzahl an Überhangmandaten } & $\begin{array}{c}\text { Vorsprung von } \\
\text { CDU/CSU } \\
\text { versus SPD }\end{array}$ & $\begin{array}{c}\text { Größe des } \\
\text { Bundestags }\end{array}$ \\
\hline 20,2 & 1,0 & 2,3 & 23,5 & 18,9 & 621,5 \\
\hline
\end{tabular}

Im Mittel erhält die CDU in den 1000 Simulationen circa 20 Überhangmandate, die gesamte Bandbreite der Werte geht von fünf bis 32. Der Mittelwert für die CSU liegt bei einem Überhangmandat, die gesamte Spannweite erstreckt sich von null bis sieben Überhangmandate, wobei null der Modalwert ist, der allein über die Hälfte der Fälle enthält. Die SPD bekommt in der Simulation im Mittel ungefähr zwei Überhangmandate, die Bandbreite der Werte reicht von null bis elf. Die Verteilungen sind in den Abbildungen 1 bis 3 grafisch dargestellt.

Der Vorsprung an Überhangmandaten von CDU und CSU gegenüber der SPD beträgt im Mittel ungefähr 19 Mandate, also etwa 3 Prozent aller Sitze im Bundestag. Allerdings sollte man bei der Interpretation nicht zu sehr auf den Mittelwert abstellen, da die Streuung nicht unerheblich ist, wie in Abbildung $4 \mathrm{zu}$ sehen ist. 

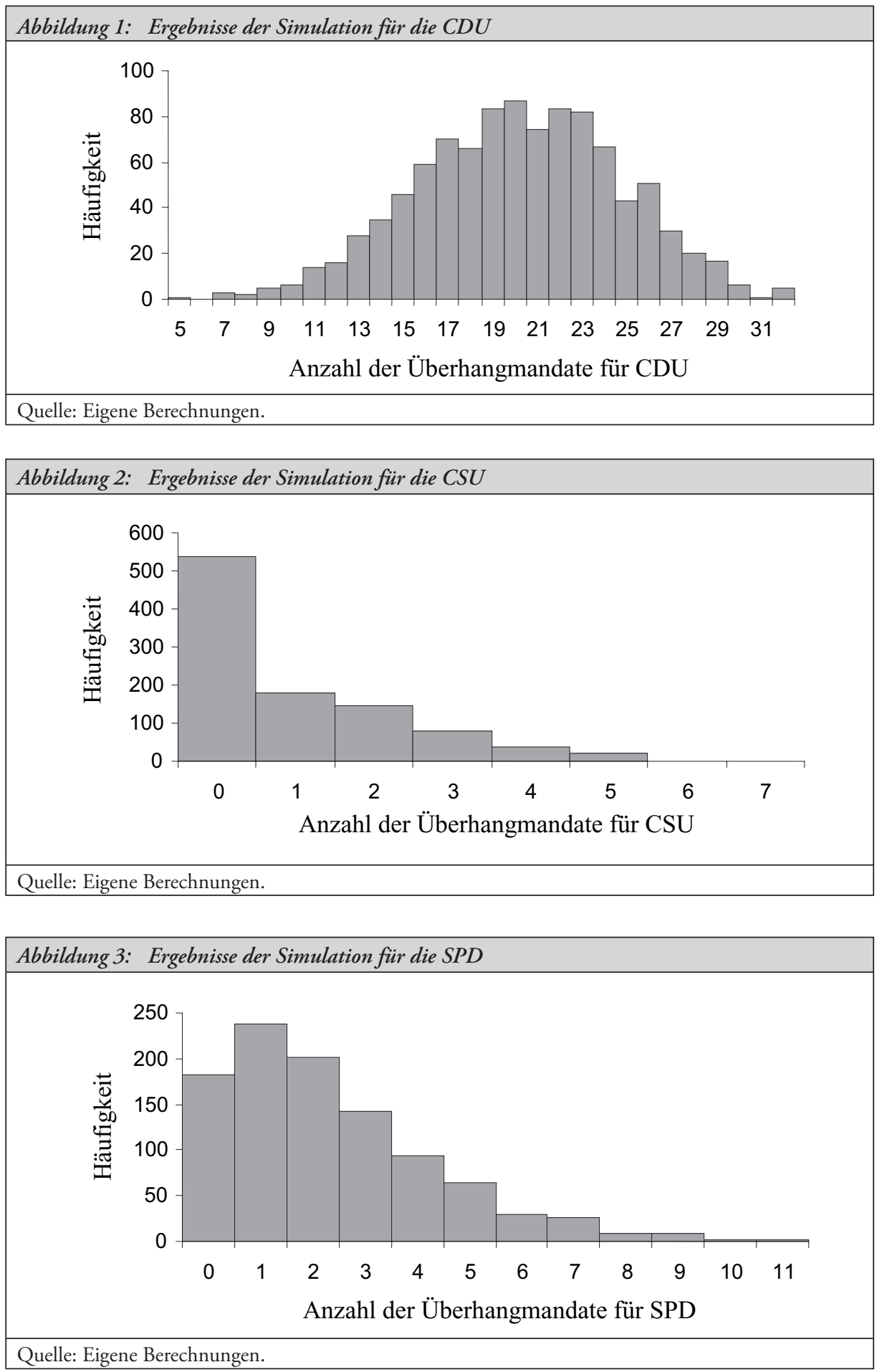


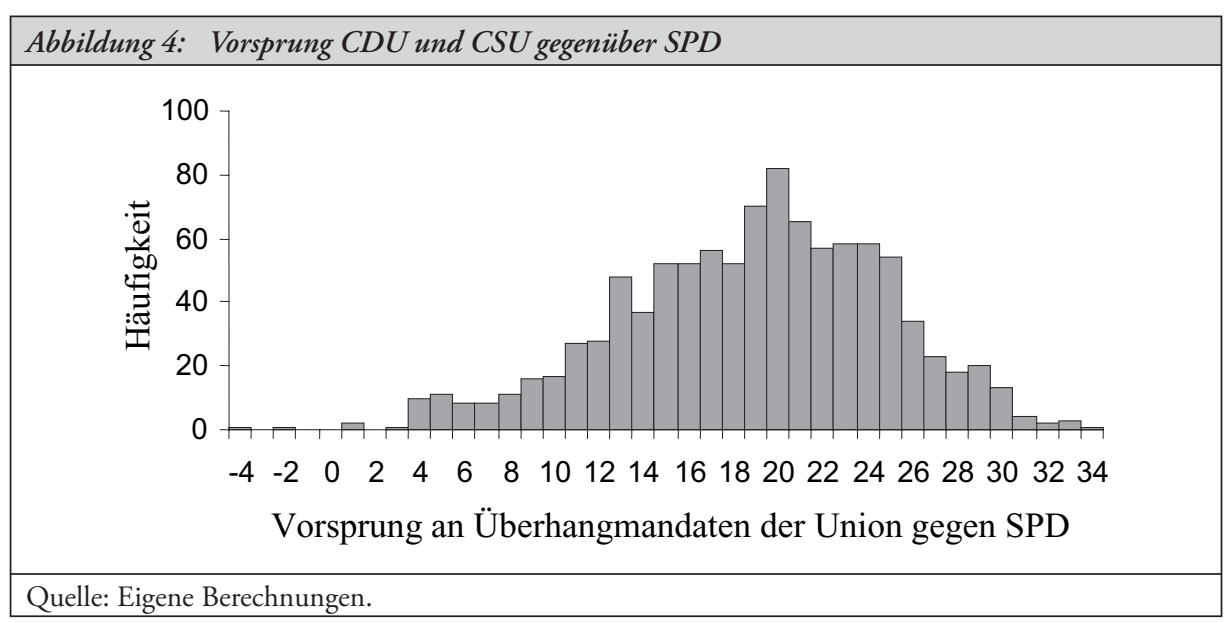

Der Bundestag wächst durch die Überhangmandate durchschnittlich um circa 24 auf 622 Sitze. Der spannendste Aspekt besteht natürlich darin, inwieweit sich das politische Ergebnis einer Wahl verändert, indem die Überhangmandate neue Koalitionsbildungsmöglichkeiten schaffen oder bestehende zerstören.

Nach den derzeitigen Umfragedaten sieht alles danach aus, dass sich für die schwarzgelbe Koalition eine Mehrheit an Sitzen auch ohne Überhangmandate einstellen könnte. In 77 Prozent der Fälle ergibt sich bei den 1000 Simulationen im Standardmodell eine Mehrheit für die schwarz-gelbe Koalition auch ohne Überhangmandate. Nimmt man diese hinzu, erhöht sich der Anteil mit einer schwarz-gelben Sitzmehrheit auf 90 Prozent. Anders ausgedrückt: Mit einer Wahrscheinlichkeit von ungefähr einem Achtel gibt es einen Wechsel der Mehrheitsverhältnisse mit der Folge, dass die CDU/CSU-FDP-Koalition aufgrund der Überhangmandate eine Mehrheit erhielte, die sie ohne diese nicht hätte. Die Überhangmandate schüfen also eine „künstliche Mehrheit“8.

In ungefähr 43 Prozent der Fälle würde es zu einer schwarz-grünen Koalition ohne Überhangmandate reichen; mit diesen vergrößerte sich dieser Anteil auf 75 Prozent. Die

8 In der Literatur wird in Bezug auf Mehrheitswahlsysteme von „manufactured majorities“ und „artificial minorities" gesprochen, vgl. Douglas W. Rae, The Political Consequences of Electoral Laws, New Haven 1967, S. 74 f. „Manufactured majorities“ sind Mehrheiten, die durch den im Mehrheitswahlsystem angelegten Verzerrungseffekt die bewusste Intention des Wahlsystems widerspiegeln, der stärksten Partei die absolute Mehrheit im Parlament zu verschaffen. „Artificial minorities" hingegen sind systemwidrig, das heißt es handelt sich dabei um verlorene Mehrheiten, die vorkommen, wenn eine Partei mit der absoluten Mehrheit an Stimmen dennoch eine Minderheit der Sitze erhielte. Bezogen auf ein Verhältniswahlsystem spreche ich daher von „künstlichen Mehrheiten“, wenn ein Zusammenschluss von Parteien die Mehrheit von Sitzen erhält, der weniger Stimmen auf sich vereinigen konnte als die übrigen Parteien. Da Überhangmandate auch im Design nicht gezielt dazu angelegt sind, manufactured majorities zu schaffen beziehungsweise deren Auftreten zu begünstigen, scheint es wenig sinnvoll von "guten“ und „schlechten“ Überhangmandaten zu sprechen, je nachdem, welche Mehrheitskoalition damit ermöglicht oder unterbunden wird. Im Gegensatz zur Logik des Mehrheitswahlsystems gibt es im Verhältniswahlsystem keine herausgehobene Bedeutung der größten Partei, die es rechtfertigen könnte, diese bewusst mit einem Bonus zu versehen. 


\begin{tabular}{|l|c|c|c|}
\hline Tabelle 4: Anteil in Prozent der Simulationen, in denen eine bestimmte Koalition eine Mehrheit \\
hat (Standardmodell)
\end{tabular}

Überhangmandate erhöhen also nicht nur die Wahrscheinlichkeit, dass die CDU/CSU eine Mehrheitskoalition mit der FDP erreichen kann, sondern sie eröffnen der Union strategische Verhandlungsspielräume für zusätzliche Koalitionsmöglichkeiten. In 38 Prozent der Fälle (ohne Überhangmandate) beziehungsweise in 71 Prozent (mit Überhangmandaten) kann sich die Union nach rechnerischer Maßgabe aussuchen, mit welchem der beiden kleineren Partner sie eine Koalition eingehen will.

Die mit der Simulation geschaffene Projektion trifft die Wirklichkeit natürlich nur so gut, wie die zugrunde gelegten Annahmen dies erlauben. Mit so genannten Sensitivitätsanalysen ${ }^{9}$ kann überprüft werden, inwieweit die Ergebnisse von den Annahmen geprägt sind. Dies wird im folgenden Abschnitt lediglich in Bezug auf einige Szenarien, die verschiedene strategische Optionen der Parteien beinhalten, durchgeführt. ${ }^{10}$

\section{Modiffkationen des Grundmodells, die die strategischen Optionen der Parteien widerspiegeln}

Im so genannten Standardmodell (vgl. Tabelle 2) wurden bestimmte Annahmen über das Splittingverhalten gemacht, die im Folgenden modifiziert werden sollen. Dabei wird weiterhin davon ausgegangen, dass die Splittingmuster von FDP- und Grünen-Anhängern relativ eingeübt und daher mehr oder weniger stabil sind. Die Annahme, dass 60 Prozent der Zweitstimmen-FDP-Wähler mit ihrer Erststimme die CDU und 60 Prozent der Zweitstimmen-Grünen-Wähler mit ihrer Erststimme die SPD wählen, soll daher beibehalten und nicht weiter modifiziert werden.

Hingegen könnte sich das Splittingverhalten der Wähler der Linken noch deutlich verändern, was - insbesondere in den neuen Bundesländern aufgrund der dort umfangreichen Ressourcen an Wählerstimmen - einen erheblichen Effekt auf die Gewinnung von Überhangmandaten ausüben würde. Einerseits sollte die spontane Neigung, die Erststimme einer anderen Partei zu geben, bei den Anhängern der Linkspartei aus naheliegenden Gründen eher gering ausfallen. Im Gegensatz zu FDP und Grünen kann Die Linke in den neuen

9 Joachim Behnke, Simulation, in: Kai-Uwe Schnapp / Nathalie Behnke / ders. (Hrsg.), Datenwelten. Datenerhebung und Datenbestände in der Politikwissenschaft, Baden-Baden 2009, S. 174 195.

10 Analysen hinsichtlich der modelleigenen Parameter, insbesondere der angenommenen Streuungen, unterbleiben hier aus Platzgründen. Es sei nur grundsätzlich erwähnt, dass die Unterschiede mit zunehmenden Standardabweichungen der Veränderungsraten zwar abnehmen, sich aber keineswegs verflüchtigen. So sinkt zum Beispiel der Vorsprung der Union gegenüber der SPD an Überhangmandaten von circa 20 auf ungefähr 15 bis 16, wenn man die Standardabweichung der Veränderungsraten zwischen den Wahlkreisen annähernd verdoppelt. 
Bundesländern Direktmandate gewinnen. 2009 stehen dafür die Chancen vermutlich wesentlich besser als je zuvor. Außerdem gibt es für Die Linke keine prä-elektorale Koalition ${ }^{11}$, das heißt es existieren keine potenziellen Partner, die ihre eindeutige Absicht erklärt hätten, mit ihr eine Koalition bilden zu wollen. Andererseits hat Die Linke selbst derzeit keinerlei Aussicht auf den Gewinn von Überhangmandaten: In keiner einzigen der 1000 Iterationen des Standardmodells entstanden für sie Überhangmandate.

Bleibt die Frage, wie hoch Die Linke den symbolischen Effekt einschätzt, einen Wahlkreis zu gewinnen. Der greifbare Nutzen von Direktmandaten ist für sie eher gering. Die Zeiten, in denen die Partei den Gewinn von Direktmandaten anstreben musste, um sich so nötigenfalls über die Grundmandatsklausel die Teilnahme an der proportionalen Sitzverrechnung zu garantieren, falls sie an der Fünfprozenthürde scheitern sollte, sind eindeutig vorüber. Auch für die personelle Besetzung im Bundestag dürfte es kaum Auswirkungen haben, ob Die Linke Direktmandate gewinnt, da erfolgreiche Kandidaten in den Wahlkreisen vermutlich in der Regel auch über die Liste einziehen würden. Aus diesen Gründen könnten Wähler der Linken in Zukunft in einem wesentlich höheren Maß mit ihrer Erststimme die SPD unterstützen, als dies bisher geschehen ist. In Tabelle 5 sind die grundlegenden Ergebnisse der Simulation in Abhängigkeit von verschiedenen Splittingquoten der Die Linke-Wähler zugunsten der SPD mit der Erststimme aufgeführt. Dabei wurde davon ausgegangen, dass die Splittingneigung in der Regel im Westen höher ausfallen dürfte zum einen, weil es dort überhaupt keine Chance auf den Gewinn eines eigenen Direktmandats gibt, zum anderen, weil ein nicht unbeträchtlicher Teil der Wähler der Linken im Westen aus ehemaligen SPD-Wählern besteht.

Es zeigt sich sehr deutlich, dass sich die Überhangmandate nicht zwangsläufig zum Vorteil der Union auswirken müssen. Je ausgeprägter das Splittingverhalten der Anhänger der Linken zugunsten der SPD, desto geringer fällt der Vorteil für die CDU/CSU aus und dreht sich schließlich sogar in einen Nachteil um. Während die Union im Standardmodell durch einen Vorsprung von 19 Überhangmandaten gegenüber der SPD ihre Siegchancen in einer schwarz-gelben Koalition von knapp 80 auf 90 Prozent zu erhöhen vermag, sinken im Extremfall - wenn die Anhänger der Linken sowohl im Westen als auch im Osten zu 60 Prozent ihre Erststimme der SPD geben - diese Chancen auf circa 60 Prozent, da die Union nun im Durchschnitt zwölf Überhangmandate weniger erhielte als die SPD.

Allerdings könnten die Wähler der Union dieser Gefahr mit einer Gegenstrategie begegnen, die sie schon 2005 erfolgreich in Dresden angewandt haben. Da schon vor der Nachwahl in Dresden klar war, dass die CDU in Sachsen in jedem Fall Überhangmandate erhalten würde, war für die Dresdner Wähler klar, dass ihre Zweitstimme für die CDU so oder so verschenkt gewesen wäre. Schätzungsweise ein Sechstel der potenziellen CDU-Sympathisanten entschied sich daher, der FDP ihre für die CDU nutzlose Zweitstimme zu geben ${ }^{12}$, um so ein zusätzliches Überhangmandat für die CDU zu schaffen und gleichzeitig mit ihrer Zweitstimme an der Gewinnung regulärer Listenmandate für die FDP mitzuwirken.

Das „Simulationsmodell mit Transfer“ arbeitet daher mit einem zusätzlichen Stimmentransfer von der CDU zur FDP. Es erscheint plausibel anzunehmen, dass 15 Prozent der

11 Vgl. Marc Debus, Pre-Electoral Alliances, Coalition Rejections, and Multiparty Governments, Baden-Baden 2007.

12 Vgl. Joachim Behnke, Strategisches Wählen bei der Nachwahl in Dresden zur Bundestagswahl 2005, in: PVS, 49. Jg. (2008), S. $695-720$. 
Zweitstimmen der CDU zur FDP transferiert werden. Dieser Wert dürfte vor dem Hintergrund der Dresdner Erfahrungen das Mobilisierungspotenzial der CDU, ihre Anhänger zu strategischem Verhalten zu bewegen, halbwegs realistisch wiedergeben. Allerdings ist davon auszugehen, dass nicht alle CDU-Wähler in allen Bundesländern sich so verhalten, sondern gezielt nur in jenen Bundesländern, die eine ersichtliche Anfälligkeit für Überhangmandate zugunsten der CDU aufweisen. Es handelt sich dabei um Baden-Württemberg, Hessen, Rheinland-Pfalz, Schleswig-Holstein, Mecklenburg-Vorpommern, Sachsen, Sachsen-Anhalt und Thüringen.

Wie in Tabelle 5 zu erkennen ist, wirkt sich der Stimmentransfer lediglich auf den Gewinn von zusätzlichen Überhangmandaten zugunsten der CDU aus, während die Zahlen für die anderen Parteien nahezu unverändert bleiben. Dies liegt daran, dass hier die Überhangmandate - im Gegensatz zum Effekt des vermehrten Splittings seitens der Anhänger der Linken - nicht durch zusätzliche, erfolgreich erkämpfte Direktmandate entstehen, sondern indem ohnehin gewonnene Direktmandate in Überhangmandate „umgewandelt“ werden, da sie nicht mehr durch genügend Zweitstimmen gedeckt sind. Die Anzahl aller Überhangmandate steigt dadurch im Mittel auf einen Bereich von über 30 und kann bei den „günstigsten“ Bedingungen, die in Tabelle 5 rechts unten zu finden sind, im konkreten Einzelfall bis zu 40 (und noch mehr) erreichen.

Während die Nutzung ihrer strategischen Optionen für die Die Linke-Wähler jedoch weitgehend kostenfrei bliebe, würde sie für die CDU deutlich schmerzhafter ausfallen. Zwar erhöht sich auf diese Weise die Gewinnchance einer schwarz-gelben Koalition, der Sitzanteil der CDU aber würde sowohl im Parlament als auch in der angestrebten Koalition abnehmen. Außerdem würde sich für die CDU die Option, auch eine Koalition mit den Grünen eingehen zu können, dramatisch schmälern.

Alle bisher vorgestellten Simulationen beruhen auf Werten von Umfragen, die Anfang Juli 2009 erhoben worden sind. Allerdings leiden Umfragen nicht nur an den üblichen Unsicherheiten bezüglich der Messung, die in diesem Simulationsmodell durch die entsprechende Streuung der Umfragewerte erfasst werden, sondern die jetzigen Umfrageergebnisse könnten sogar aus systematischen Gründen relativ weit vom tatsächlichen Stimmungsbild im Juli und erst recht vom Ergebnis der Wahl im September entfernt sein. So lag ja auch 2005 die SPD zum Beispiel bei der Forschungsgruppe Wahlen und bei Infratest Dimap drei Monate vor der Wahl in den Umfragen um mehr als 15 Prozentpunkte und unmittelbar vor der Wahl noch um acht Punkte hinter der Union zurück. Dieser Vorsprung fiel dann gemäß Joschka Fischers Prophezeiung im Bundestag bei der Wahl „wie ein Soufflé" auf einen Prozentpunkt zusammen. Zweifel, inwieweit die derzeitigen Umfrageergebnisse den tatsächlichen Wahlausgang auch nur annähernd genau voraussagen können, sind also keineswegs unangemessen. ${ }^{13}$

Es bleibt aber eine unbestrittene Tatsache, dass die SPD in den Umfragen im Juli katastrophale Werte erzielt hat, die vermutlich teilweise noch unter den veröffentlichten liegen. Eine Aufholjagd im gleichen Ausmaß wie 2005 ist allein aus einem Grund unwahrscheinlich: 2005 hatte die SPD mit Gerhard Schröder den populäreren Kanzlerkandidaten, 2009 hingen liegt Angela Merkel in der Kandidatenfrage eindeutig weit vor Frank-Walter Steinmeier. Daher wird den weiteren Simulationsszenarien zugrunde gelegt, dass die beiden

13 Zur methodischen Kritik an Umfragen als Wahlprognosen vgl. Fritz Ulmer, Wahlprognosen und Meinungsumfragen, in: Bild der Wissenschaft, 24. Jg. (1987), H. 1, S. 88 - 100, S. 89 ff. 


\begin{tabular}{|c|c|c|c|c|c|}
\hline \multicolumn{6}{|c|}{ Tabelle 5: Ergebnisse der modifizierten Simulationsmodelle } \\
\hline & & \multicolumn{4}{|c|}{$\begin{array}{l}\text { Splittingverhalten der Zweitstimmen-Die Linke-Wähler } \\
\text { zugunsten der SPD (mit Erststimme) }\end{array}$} \\
\hline & & $\begin{array}{l}\text { West: } 25 \% \\
\text { Ost: } 0 \%\end{array}$ & $\begin{array}{l}\text { West: } 40 \% \\
\text { Ost: } 20 \%\end{array}$ & $\begin{array}{l}\text { West: } 50 \% \\
\text { Ost: } 40 \%\end{array}$ & $\begin{array}{l}\text { West: } 60 \% \\
\text { Ost: } 60 \%\end{array}$ \\
\hline \multirow{9}{*}{ Grundmodell } & \multicolumn{5}{|l|}{ Überhangmandate für... } \\
\hline & $\mathrm{CDU}$ & 20,2 & 14,4 & 10,3 & 7,6 \\
\hline & CSU & 1,0 & 1,0 & 0,9 & 0,9 \\
\hline & SPD & 2,3 & 7,0 & 14,2 & 20,8 \\
\hline & CDU+CSU-SPD & 18,9 & 8,5 & $-3,0$ & $-12,3$ \\
\hline & \multicolumn{5}{|c|}{ Gewinnwahrscheinlichkeit für Schwarz-Gelb } \\
\hline & ohne Überhangmandate & 77,3 & 79,8 & 79,5 & 80,2 \\
\hline & mit Überhangmandaten & 90,4 & 84,7 & 71,6 & 59,0 \\
\hline & $\begin{array}{l}\Delta \text { Gewinnwahrscheinlich- } \\
\text { keit für Schwarz-Gelb }\end{array}$ & $+13,1$ & $+4,9$ & $-7,9$ & $-21,2$ \\
\hline \multirow{9}{*}{$\begin{array}{l}\text { Grundmodell } \\
\text { mit Transfer }\end{array}$} & \multicolumn{5}{|l|}{ Überhangmandate für ... } \\
\hline & $\mathrm{CDU}$ & 33,4 & 26,3 & 21,2 & 17,5 \\
\hline & CSU & 1,1 & 1,0 & 1,0 & 0,8 \\
\hline & SPD & 2,3 & 7,0 & 14,3 & 20,8 \\
\hline & CDU+CSU-SPD & 32,1 & 20,2 & 7,9 & $-2,4$ \\
\hline & \multicolumn{5}{|c|}{ Gewinnwahrscheinlichkeit für Schwarz-Gelb } \\
\hline & ohne Überhangmandate & 79,5 & 78,2 & 79,6 & 79,7 \\
\hline & mit Überhangmandaten & 95,9 & 90 & 81,3 & 71,6 \\
\hline & $\begin{array}{l}\Delta \text { Gewinnwahrscheinlich- } \\
\text { keit für Schwarz-Gelb }\end{array}$ & $+16,4$ & $+11,8$ & $+1,7$ & $-8,1$ \\
\hline
\end{tabular}

\begin{tabular}{|cccccc|}
\hline \multicolumn{7}{|l|}{ Tabelle 6: Modellannahmen für Simulation bei, „engem “ Szenario } \\
\hline \multicolumn{4}{|l|}{ Angenommener globaler Trend der Veränderung von } & 2005 nach 2009 in Prozentpunkten für die Parteien \\
CDU & CSU & SPD & FDP & Grüne & Die Linke \\
+1 & -5 & -5 & +2 & +2 & +1 \\
\hline
\end{tabular}

Lager zwar noch enger zusammenrücken, als es in den Umfragen derzeit der Fall ist, die CDU aber weiterhin einen klaren Vorteil gegenüber der SPD behält. Die angenommenen Trends für dieses Szenario eines „engen Wahlausgangs“ sind in Tabelle 6 wiedergegeben.

Der wesentliche Unterschied zum bisherigen Modell besteht darin, dass die SPD nun „nur“ noch fünf Prozentpunkte verliert. FDP und Grüne legen etwas weniger zu als im Originalmodell, und die CSU verliert statt zwei nun fünf Prozentpunkte.

In Tabelle 7 sind die Ergebnisse der entsprechenden Simulationen aufgeführt. Im Standardmodell, bei dem die FDP- und Grünen-Zweitstimmenwähler zu 60 Prozent ihre Erststimme jeweils der CDU/CSU beziehungsweise der SPD geben und 25 Prozent der Anhänger der Linken die SPD im Westen mit ihrer Erststimme unterstützen, während sie dies im Osten ganz und gar unterlassen, fallen aufgrund des ausgewogeneren Wettbewerbs nun insgesamt „nur“ noch 17 Überhangmandate an. Der Vorteil der Union gegenüber der SPD 


\begin{tabular}{|c|c|c|c|c|c|}
\hline & & \multicolumn{4}{|c|}{$\begin{array}{l}\text { Splittingverhalten der Zweitstimmen-Die Linke-Wäh- } \\
\text { ler zugunsten der SPD (mit Erststimme) }\end{array}$} \\
\hline & & $\begin{array}{l}\text { West: } 25 \% \\
\text { Ost: } 0 \%\end{array}$ & $\begin{array}{l}\text { West: } 40 \% \\
\text { Ost: } 20 \%\end{array}$ & $\begin{array}{l}\text { West: } 50 \% \\
\text { Ost: } 40 \%\end{array}$ & $\begin{array}{l}\text { West: } 60 \% \\
\text { Ost: } 60 \%\end{array}$ \\
\hline \multirow{9}{*}{ Grundmodell } & \multicolumn{5}{|l|}{ Überhangmandate für ... } \\
\hline & $\mathrm{CDU}$ & 11,5 & 7,0 & 4,5 & 2,9 \\
\hline & $\mathrm{CSU}$ & 1,8 & 1,5 & 1,4 & 1,2 \\
\hline & SPD & 4,2 & 10,2 & 16,4 & 21,2 \\
\hline & CDU+CSU-SPD & 9,1 & $-1,8$ & $-10,4$ & $-17,1$ \\
\hline & \multicolumn{5}{|c|}{ Gewinnwahrscheinlichkeit für Schwarz-Gelb } \\
\hline & ohne Überhangmandate & 24,9 & 25,8 & 25,9 & 27,3 \\
\hline & mit Überhangmandaten & 44,6 & 29,2 & 18,7 & 12,9 \\
\hline & $\begin{array}{l}\Delta \text { Gewinnwahrscheinlichkeit } \\
\text { für Schwarz-Gelb }\end{array}$ & $+19,7$ & $+3,4$ & $-7,2$ & $-14,4$ \\
\hline \multirow{9}{*}{$\begin{array}{l}\text { Grundmodell } \\
\text { mit Transfer }\end{array}$} & \multicolumn{5}{|l|}{ Überhangmandate für ... } \\
\hline & $\mathrm{CDU}$ & 22,0 & 16,0 & 12,1 & 9,6 \\
\hline & $\mathrm{CSU}$ & 1,8 & 1,5 & 1,3 & 1,2 \\
\hline & SPD & 4,2 & 10,2 & 16,6 & 21,0 \\
\hline & CDU+CSU-SPD & 19,6 & 7,3 & $-3,2$ & $-10,2$ \\
\hline & \multicolumn{5}{|c|}{ Gewinnwahrscheinlichkeit für Schwarz-Gelb } \\
\hline & ohne Überhangmandate & 25,2 & 27,0 & 23,0 & 28,7 \\
\hline & mit Überhangmandaten & 58,6 & 41,4 & 25,4 & 22,3 \\
\hline & $\begin{array}{l}\Delta \text { Gewinnwahrscheinlichkeit } \\
\text { für Schwarz-Gelb }\end{array}$ & $+33,4$ & $+14,4$ & $+2,4$ & $-6,4$ \\
\hline
\end{tabular}

halbiert sich auf neun Überhangmandate. Der Effekt in Form wachsender Gewinnwahrscheinlichkeit einer schwarz-gelben Koalition nimmt allerdings sogar zu. Mit Hilfe der Überhangmandate erhöht sich die Chance einer schwarz-gelben Mehrheit an Sitzen um circa 20 Prozentpunkte, also in einem Fünftel der Fälle verhelfen die Überhangmandate der schwarz-gelben Koalition zu einer Mehrheit, die sie ohne Überhangmandate nicht hätte. Dies ist vor allem mit dem niedrigeren Ausgangsniveau zu erklären. Ohne Überhangmandate würde das schwarz-gelbe Bündnis im „engen“ Szenario nämlich nur noch mit einer Wahrscheinlichkeit von 25 Prozent eine Mehrheit an Sitzen erringen. Durch Stimmentransfer, also wenn circa 15 Prozent der CDU-Anhänger ihre Zweitstimme der FDP gäben, erhöhte sich die Gewinnwahrscheinlichkeit für Schwarz-Gelb auf knapp 60 Prozent.

Aufgrund des niedrigen Ausgangsniveaus wachsen die Gewinnchancen der schwarz-gelben Koalition sogar dann noch, wenn sie im Mittel weniger durch Überhangmandate profitiert als die SPD. Obwohl im zweiten Szenario in der oberen Hälfte die SPD im Schnitt 1,8 Überhangmandate mehr erhält als die Union, erhöht sich die Gewinnwahrscheinlichkeit einer schwarz-gelben Koalition um etwas mehr als drei Prozentpunkte. Dies ist dadurch zu erklären, dass in 36 Prozent der Fälle die Union mehr Überhangmandate erhält, während in den verbleibenden 64 Prozent der Vorteil bei der SPD liegt. Der Anteil der Fälle, in denen die Union von den Überhangmandaten profitiert, ist also um zehn Prozent- 
punkte höher als der Anteil jener, in denen sie „sowieso“ gewonnen hätte. Daher erhöhen sich ihre Gewinnchancen sogar dann, wenn sie im Mittel weniger Überhangmandate erhält.

Auch im „engen“ Szenario gilt natürlich, dass es vor allem am Splittingverhalten der Die Linke-Wähler hängt, für welche Seite sich die Überhangmandate letzten Endes als Vorteil auswirken. Wenn diese zu 60 Prozent mit ihrer Erststimme die SPD wählten, verringerte sich die Wahrscheinlichkeit für eine schwarz-gelbe Mehrheit auf magere 13 Prozent, solange die Union den Stimmentransfer unterlässt. Durch Stimmentransfer zur FDP könnte die Union allerdings ihre Gewinnchance noch einmal um circa zehn Prozentpunkte anheben.

\section{Fazit: Reform des Wahlrechts dringend geboten}

Unter der Voraussetzung, dass der Trend, wie er sich in den Meinungsumfragen im Juli 2009 darstellt, die tatsächlichen Wählerabsichten halbwegs zuverlässig abbildet, kann man davon ausgehen, dass die Wahrscheinlichkeit, dass es für eine schwarz-gelbe Mehrheit im September reicht, bei ungefähr 80 Prozent liegt. Würden sich alle Wähler annähernd so verhalten, wie sie es bei den letzten Bundestagswahlen getan haben, erhöht sich diese Wahrscheinlichkeit aufgrund der Überhangmandate auf etwa 90 Prozent. Die erwartete Anzahl der Überhangmandate liegt bei ungefähr 23. In 94 Prozent der Fälle der Simulation ergibt sich ein Wert von mehr als 16 Überhangmandaten; es darf also als ziemlich sicher gelten, dass es unter den genannten Bedingungen bei der Bundestagswahl zu einem neuen Spitzenwert an Überhangmandaten kommen wird. Dies erhöht den Anreiz zu strategischem Verhalten in einem ebenfalls bisher nicht gekannten Maße. Die Möglichkeit, durch Stimmensplitting Überhangmandate zu schaffen, indem die Anhänger einer großen Partei ihre Zweitstimmen einer kleinen Partei geben, bei gleichzeitiger „normaler“ Unterstützung der großen Partei durch die Erststimmen der kleinen Partei, ist seit den 50er Jahren bekannt ${ }^{14}$, dürfte aber jetzt, nicht zuletzt mit Hilfe des Internets, eine Wirkung ganz neuer Dimensionen entfalten. Das Unbehagen der potenziellen zukünftigen Oppositionsparteien, „denken sie an Überhangmandate in der Nacht“, ist daher gut verständlich und dürfte ihnen nicht nur den Schlaf rauben, sondern ganz konkret auch die Chance auf eigene Regierungsbeteiligung.

In der öffentlichen Wahrnehmung - und gewiss gefördert durch die Parteien der Opposition - dürfte die Legitimation einer Regierung, die sich auf eine Parlamentsmehrheit stützen würde, die nur aufgrund von Überhangmandaten besteht, auf wackligen Füßen stehen. Jedoch wie auch immer die Wahl im September ausgehen mag, der Bundestag ist in jedem Fall dazu aufgerufen, größere Änderungen am Wahlgesetz vorzunehmen. Er wäre dabei klug beraten, sich nicht ausschließlich auf den verfassungsrechtlichen Aspekt zu konzentrie-

14 Vgl. Hans Meyer, Wahlsystem und Verfassungsordnung. Bedeutung und Grenzen wahlsystematischer Gestaltung nach dem Grundgesetz, Frankfurt am Main 1973; Harald Schoen, Stimmensplitting bei Bundestagswahlen: eine Form taktischer Wahlentscheidung?, in: ZParl, 29. Jg. (1998), H. 2, S. 223 - 244; ders., Appelle zu taktischem Wahlverhalten - effektive Werbung oder verfehlte Wahlkampfrhetorik?, in: Jürgen Falter / Oscar W. Gabriel / Hans Rattinger (Hrsg.), Wirklich ein Volk? Die politischen Orientierungen von Ost- und Westdeutschen im Vergleich, Opladen 2000, S. 639 - 672, S. 641 ff.; Joachim Behnke, Überhangmandate: Ein (behebbarer) Makel im institutionellen Design des Wahlsystems, in: ZPol, 13. Jg. (2003), H. 3, S. 1235 - 1269. 
ren. Eine Änderung, die nur das negative Stimmgewicht, nicht jedoch die Überhangmandate beseitigte, wäre lediglich eine kosmetische Maßnahme, die den Erfordernissen, denen ein Wahlgesetz zu genügen hat, nicht gerecht würde. Ziel einer Reform muss es sein, die politische Funktion einer Wahl sicherzustellen, und das heißt die unstrittige Legitimation der durch die Wahl bestellten Regierung bei gleichzeitiger Verfolgung einer bestimmten Art von Repräsentationsziel ${ }^{15}$. Diese politische Funktion kann aber nur erfüllt werden, wenn die unheilvolle Rolle, die die Überhangmandate seit der deutschen Einheit spielen, bald ihre letzte Aufführung erlebt.

Es existieren seit geraumer Zeit mehrere Vorschläge, wie die Überhangmandate beseitigt, die Wahrscheinlichkeit ihres Auftretens gesenkt oder ihre Auswirkungen auf die Sitzverteilung neutralisiert werden könnten ${ }^{16}$. Eine Verrechnung von überschüssigen Direktmandaten in einem Bundesland mit Listenmandaten derselben Partei in einem anderen Bundesland, wie im Gesetzentwurf der Grünen vorgesehen, würde das Problem zumindest entschärfen. Allerdings würden eventuelle Überhangmandate der CSU nicht verrechnet, und es könnte sogar der Anreiz entstehen, analog zur CSU regionale Repräsentanzen zu gründen. Am sinnvollsten und effektivsten wäre daher eine Kombination mehrerer Elemente, wie zum Beispiel die Verrechnung von Landeslisten plus Ausgleichsmandate für verbleibende Überschüsse. Um die Entstehung von Überhangmandaten von vornherein zu erschweren, wäre es auch möglich, den Anteil der Direktmandate an allen regulären Mandaten von 50 etwa auf 40 Prozent zu verringern oder Zweimann-Wahlkreise einzurichten. Radikaler wäre die Abschaffung des Zweistimmensystems, so dass wie 1949 mit den dann einzigen Stimmen einerseits die Wahlkreiskandidaten gewählt werden und andererseits die Gesamtzahl der Sitze für eine Partei proportional zur Summe aller erhaltenen Stimmen berechnet wird.

Diese Kombination mehrerer Elemente würde zwar die Komplexität des Wahlsystems noch einmal geringfügig erhöhen, aber wir sollten uns sowieso endgültig von der Illusion verabschieden, es könne ein transparentes Wahlsystem geschaffen werden, ohne dafür andere Nachteile in Kauf nehmen zu müssen. Es verhält sich beim Wahlsystem nicht anders als beim Steuersystem: Komplexität an sich ist keineswegs unbedingt schlecht, sondern häufig die notwendige Folge eines Regelwerks, das vielen Bedingungen genügen soll, nicht zuletzt einigen grundlegenden Gerechtigkeits- und Fairnesserfordernissen. Der Wunsch nach Einfachheit ist oft ein schlechter Ratgeber. Es wäre schwerlich etwas gewonnen, wenn der einzelne Bürger die endgültige Sitzverteilung bei vorliegendem Wahlergebnis auf einem Bierdeckel ausrechnen könnte. Es ist nicht nötig, dass jeder die komplizierte Mechanik des Wahlsystems versteht. Nicht jede einzelne Regel selbst muss transparent sein, aber das Regelwerk muss sich an Prinzipien orientieren, die einsichtig sind und von der überwiegenden Mehrheit der Bevölkerung als sinnvoll und richtig (an)erkannt werden.

15 Vgl. Dieter Nohlen, Wahlrecht und Parteiensystem, Opladen 2009; ders., Erfolgswertgleichheit als fixe Idee oder: Zurück zu Weimar? Zum Urteil des Bundesverfassungsgerichts über das Bundeswahlgesetz vom 3. Juli 2008, in: ZParl, 40. Jg. (2009), H. 1, S. 179 - 195.

16 Vgl. hierzu als Übersicht Joachim Behnke, Die Bombe tickt weiter: Das immer noch existierende Problem der Überhangmandate und mögliche Lösungen, in: Jürgen W. Falter / Oscar W. Gabriel / Bernhard Weßels (Hrsg.), Wahlen und Wähler. Analysen aus Anlass der Bundestagswahl 2002, Wiesbaden 2005, S. 459 - 483; ders., a.a.O. (Fn. 2), S. 196 ff.; siehe auch Ossip Fürnberg I Danko Knothe, a.a.O. (Fn. 2), S. $71 \mathrm{ff}$. 